\title{
Acute Dengue Infection in the Western Terai Region of Nepal
}

\author{
Pun R, ${ }^{1}$ Pant KP, ${ }^{1}$ Bhatta DR, ${ }^{1}$ Pandey BD ${ }^{2}$ \\ ${ }^{1}$ Central Department of Microbiology, Tribhuvan University, Kathmandu. ${ }^{2}$ Sukraraj Tropical and Infectious Diseases Hospital, \\ Kathmandu, Nepal and Everest International Clinic and Research Center, Kalanki, Kathmandu
}

\section{ABSTRACT}

Introduction: Dengue fever is an emerging mosquito borne disease in Nepal claiming substantial morbidity and mortality. The objective of the study was to find out frequency of acute dengue infection in patients from the hospitals of the western Nepal.

Methods: The study was conducted between August 2007 and July 2008 in patients visiting hospitals of the western terai of Nepal with chief complains of fever. The sero-diagnosis of acute dengue infection was determined by enzyme linked immunosorbent assay among 239 patients visiting Lumbini Zonal Hospital, Butwal; Bheri Zonal Hospital, Nepalgunj; Bardiya District Hospital, Bardiya and Mahakali Zonal Hospital, Mahendranagar.

Results: The anti-dengue IgM positivity was $29.3 \%$. There was slight male preponderance with a male to female ratio of 1.2:1. Out of the total positive cases, the highest positive cases (75.7 \%) were from the age group 15 - 50 years followed by $<15$ years old $(15.7 \%)$. Out of four hospitals, the highest positive cases $(54.3 \%)$ were in Lumbini Zonal Hospital, Butwal. The age and gender were independent predictors to dengue virus infection. The highest numbers of dengue positive cases were in October (52.6 \%). The association between dengue disease and the month was statistically significant.

Conclusions: The dengue positivity was estimated in acute patients from hospitals of western Nepal by enzyme immunoassay. Therefore, the serological marker can be used to diagnose acute patients of dengue during outbreaks.

Keywords: dengue, ELISA, post monsoon, sero-diagnosis

\section{INTRODUCTION}

Dengue is an acute febrile illness caused by a virus that is transmitted from humans to humans via species of infected Aedes aegypti mosquito. Four dengue virus (DENV) serotypes, i.e. DEN1-4, belonging to the genus flavivirus, are responsible for the disease. It manifests as dengue fever (DF), dengue hemorrhagic fever (DHF) and dengue shock syndrome (DSS). ${ }^{1}$ Specific symptoms vary according to the severity of dengue. ${ }^{2}$ Previous studies have shown that all the serotypes of DENV are found in Nepal. ${ }^{3,4,5}$ Nepal reported indigenous transmission of dengue cases $^{3}$, although DF had already been observed in foreign visitors earlier. ${ }^{3,6}$ Because of the open border, Nepal is vulnerable to more serious consequences of DENV infection as infected people can

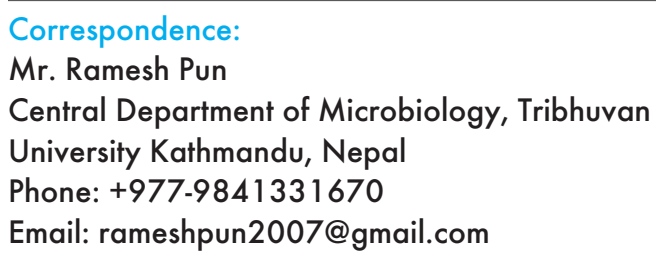


easily come across and the virus might be transmitted by the vector, which is already evident in several locations of the terai region. The aim of the study was to determine the frequency of acute dengue infection using the serological method. The present study would be helpful by providing information on the epidemiology of the disease in the western terai region of Nepal.

\section{METHODS}

This was a cross-sectional study conducted between August 2007 and July 2008 in which samples were collected during the post-monsoon period (August November 2007). The serum samples were collected from Mahakali Zonal Hospital, Mahendranagar; Bardiya District Hospital, Bardiya; Bheri Zonal Hospital, Nepalgunj and Lumbini Zonal Hospital, Butwal. Patients with high fever and clinical symptoms suspected of DENV infection (according to $\mathrm{WHO}$ guideline) ${ }^{7}$ visiting the hospitals were included in the study, while patients infected with malaria and enteric fever were excluded. Similarly, subjects with previous Japanese Encephalitis immunizations were also excluded from the study. Patients' personal details about the symptoms and age, sex, etc, were obtained through a questionnaire method by direct interview after taking the consent.

Dengue antibodies were measured with enzyme linked immunosorbent assay, i.e. IgM capture ELISA (PanBio, Australia), and results interpreted according to the manufacturer's instructions. The cut-off value was obtained by multiplication of the average absorbance of calibrators and calibration factor (provided in the kit, i.e. 1).

The collected data was analyzed to find out the age, sex, hospital and month wise distribution of the cases. Chi square test was used as a statistical tool. The statistics was done using Win Pepi software version 7.9, 2008

\section{RESULTS}

Among 239 suspected dengue cases investigated during the study period, $132(55.2 \%)$ were males and 107 (44.8\%) were females. The patients under investigation were of the age range $1-78$ years. The highest numbers of cases $(175,73.2 \%)$ were from the age range of 15 - 50 years followed by 35 cases $(14.6 \%)$ above 50 years and 29 cases $(12.1 \%)$ below 15 years. The highest numbers of cases $(n=118)$ were from Lumbini Zonal Hospital, Butwal followed by Mahakali Zonal Hospital, Mahendranagar ( $n=54$ ), Bardiya District Hospital, Bardiya $(n=39)$ and Bheri Zonal Hospital, Nepalgunj $(n=28)$ (Table 1).

The dengue positivity was $29.3 \%$ (70/239). Out of the total 70 positive cases, the highest numbers of positive cases were in the age group 15 - 50 years and the highest numbers of positive cases were in Lumbini Zonal Hospital, Butwal. The ratio of dengue positive cases in male to female was 1.1:1 (37:33). The association of the disease with age $(P=0.2)$ and sex $(P=0.6)$ was statistically insignificant (Table 2). Among four months, the highest number of positive cases 37 (52.9\%) were in October. Likewise, 17 (24.3\%) in November, 10 $(14.3 \%)$ in September and $6(8.6 \%)$ in August (Fig 1). The association between the month and the occurrence of disease was statistically significant $(P=0.01)$.

Table 1. Distribution of the subjects by sex, age and hospitals

\begin{tabular}{ll}
\hline Samples collected & $\mathbf{2 3 9}$ \\
\hline Male:female ratio & $1.2: 1(132: 107)$ \\
\hline Age range (years) & \\
$<15$ & 29 \\
$15-50$ & 175 \\
$>50$ & 35 \\
Hospitals & \\
LZH & 118 \\
MZH & 54 \\
\hline BDH & 39 \\
BZH & 28 \\
\hline
\end{tabular}

LZH: Lumbini Zonal Hospital, MZH: Mahakali Zonal Hospital, BDH: Bardiya District Hospital, BZH: Bheri Zonal Hospital.

\section{Table 2. Distribution of total positive cases}

\begin{tabular}{llllllllll}
\hline & LZH & & BZH & & BDH & MZH \\
& Male & Female & Male & Female & Male & Female & Male & Female \\
\hline$<15$ & 1 & 3 & 0 & 0 & 4 & 3 & 0 & 0 & 11 \\
$15-50$ & 16 & 13 & 2 & 1 & 9 & 9 & 1 & 2 & 53 \\
$>50$ & 3 & 2 & 1 & 0 & 0 & 0 & 0 & 0 & 6 \\
\hline Total & 20 & 18 & 3 & 1 & 13 & 12 & 1 & 2 & 70 \\
\hline
\end{tabular}


Pun et al. Acute Dengue Infection in the Western Terai Region of Nepal

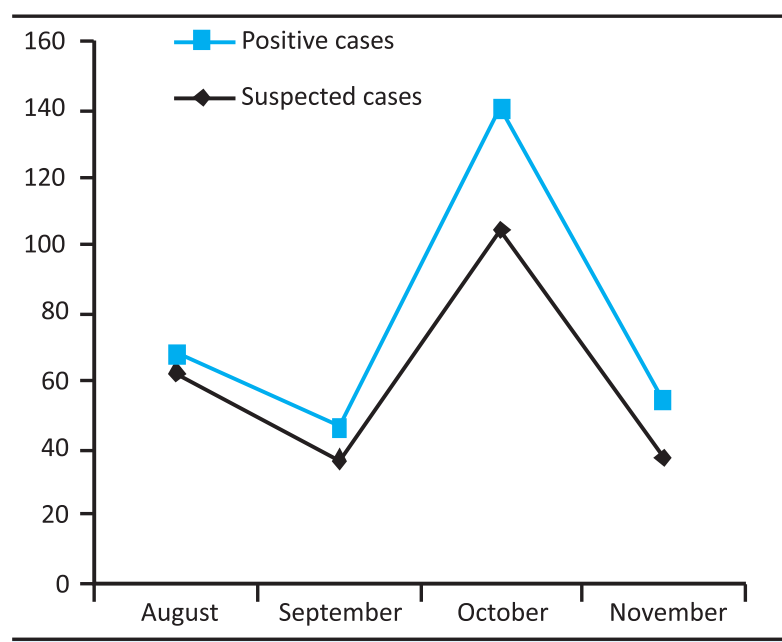

Figure 1. Month wise distribution of dengue cases.

\section{DISCUSSION}

A major proportion of the study subjects had been exposed to DENV as reflected by the increasing seropositivity of DENV infection. Information on the seroepidemiology of DENV specific IgM among Nepalese patients would provide a clue to the extent of DENV infection in Nepal. In this study, $29.3 \%$ were found to have been suffering from acute dengue infection. However, DHF and DSS cases were completely absent. The result coincides with a previous study carried out in Nepal. ${ }^{8}$

The change in demography and society due to urbanization, migration, transportation, deforestation, ecology, etc, creates an environment that helps in the emergence of dengue. ${ }^{9}$ Moreover, dengue has already been evident in neighbouring countries - China and India. The Aedes vector can easily come across the territory because of modern transportation, although the vector is already found in Nepal. The open border in the terai region also increases the transmission of the disease as infected people can come across with the vector and transmit the disease. These might be the reasons for the higher positivity of dengue in the study.

The study was based on the commonly used ELISA test (99\% sensitivity and 84.4 specificity), which also detects cross-reacting antibodies to other flavivirus, such as Japanese encephalitis virus (JEV). ${ }^{10}, 11$ The false positive results might be minimal because IgM capture ELISA is highly specific as only $10.7 \%$ DENV infection cross-reacted with JEV antigen. ${ }^{12}$ Subjects with previous JE immunizations were excluded from the study; therefore, false positive results because of cross reactivity were minimal.

The age wise distribution showed that the highest positive dengue cases were in the age group of $15-50$ years which constitutes $75.7 \%$ of the total positive cases followed by the age group $<15$ years comprising $15.7 \%$ and $8.6 \%$ in the $>50$ years age group. The result is in harmony with the data obtained in the outbreak of dengue in Nepal in the year 2006 in which the highest dengue positive cases were recorded in the age group greater than 15 years. $^{3}$

The reason for the lower number of isolates in the younger age group could be due to the improper clinical selection of cases. Dengue fever in a younger age group manifests as rather an undifferentiated illness, such as a upper respiratory like infection accompanied by headache and mild gastrointestinal complaints. The disease in adults is severe enough that patients feel sick and demand medical attention.

Out of 70 positive cases, the highest numbers of positive cases $(37,52.9 \%)$ were in October followed by November $(17,24.3 \%)$. The result is consistent with the data obtained during an outbreak of dengue in Nepal in which $75 \%$ of DF cases were reported in October and a few positive cases in September and November. ${ }^{3}$

The cases coincide mainly with the post monsoon period of subnormal rainfall because the relative prevalence and distribution of Ae. aegypti larval indices are highest during the post monsoon period. Heavy rainfall subsequently leads to a decrease in temperature during the later part of the monsoon period. The temperature remains almost constant and subsequent rainfall further leads to a decrease in the temperature. The result is an increase in relative humidity and the abundant stocks of fresh water reservoirs generated due to rain develop optimum conditions for mass breeding and propagation of the vector and transmission of the virus. ${ }^{13}$

The highest numbers of positive cases were in Lumbini Zonal Hospital, Butwal. The comparatively higher positive cases might be due to the abundance of the vector, the Aedes species mosquitoes, and the circulation of multiple dengue viral strains. Butwal is one of the busiest stations from where people go to other parts of the country as well as to India and vice versa. So, DEN infected people might come across with the vector and transmit the disease.

The cross reactivity to JEV was not performed. Additionally, paired serum samples were not taken during laboratory tests. These remained the limitations of the study.

\section{CONCLUSIONS}

The positivity of dengue was estimated in acute patients from hospitals of western Nepal by IgM capture ELISA and the most affected group was the $15-50$ years. 
Pun et al. Acute Dengue Infection in the Western Terai Region of Nepal

Thus, ELISA can be used to diagnose acute patients of dengue and people of all age groups should be educated about the mode of disease transmission and prevention. Artificial collections of water should be discouraged in order to prevent breeding of Aedes mosquitoes and enhanced vector control programmes should be commenced.

\section{ACKNOWLEDGEMENTS}

The authors thank Everest International Clinic and Research Centre (EICRC), different participating hospitals, Kiran Pandey and Dr. Durga Dutta Joshi for their cooperation.

\section{REFERENCES}

1. Gubler DJ. Dengue and dengue hemorrhagic fever. J Clin Microbiol Rev 1998; 11:480-96.

2. Schreiber KV. An investigation of relationships between climate and dengue using a water budgeting techniques. J Int Biomet 2001;45:81-89.

3. WHO/SEARO. Outbreak investigation of DF in Nepal 2006 (http://www.searo.who.int/LinkFiles/Dengue_dengue_ Nepal.pdf, Accessed 25 January 2009).

4. Takasaki T, Kotaki A, Nishimura K, Sato Y, Tokuda A, Lim $\mathrm{CK}$, et al. Dengue virus type 2 isolated from an imported dengue patient in Japan: first isolation of dengue virus from Nepal. J Travel Med 2008;15:46-49.

5. Pandey BD. Dengue emerging in Nepal. J Emerg Infect Dis 2008;14:514-5.

6. Pandey BD, Rai SK, Morita K and Kurane I. First case of dengue in Nepal. J Nepal Med College 2004;6:157-59.

7. World Health Organization. Dengue hemorrhagic fever: diagnosis, treatment and control, 2nd Ed. Geneva, World Health Organization, 1997.

8. Sah O. Sero-epidemiological and molecular study of dengue viruses in terai region of Nepal. Masters dissertation, Tribhuvan university. 2008.
9. Gubler DJ. Dengue and dengue hemorrhagic fever: its history and resurgence as a global public health problem. CAB International, London, United Kingdom. 1997.

10. World Health Organization. Evaluation of commercially available anti-dengue virus immunoglobulin $\mathrm{M}$ tests. Diagnostics Evaluation Series No. 3. 2009. (http:/ /apps.who. int/tdr/publications/tdr-research-publications/diagnosticsevaluation-3/pdf/diagnostics-evaluation-3.pdf, Accessed 27 February 2010).

11. Innis BL, Nisalak A, Nimmannitya S, Kusalerdchariya S, Chongswasdi V, Suntayakorn S, et al. An enzyme-linked immunosorbent assay to characterize dengue infections where dengue and Japanese encephalitis co-circulate. J Am Trop Med Hyg 1989;40:418-27.

12. Sherchand JB, Pandey BD, Haruki K, Jimba M. Sero-diagnosis of Japanese encephalitis and dengue virus infection from clinically suspected patients of Nepal. J Inst Med 2001;23:8185

13. Chakravorti A and Kumaria R. Eco-epidemiological analysis of dengue infection during an outbreak of dengue fever, India. J Viro 2005;2:1-6. 